\title{
The 2015 Young Innovators of Cellular and Molecular Bioengineering
}

It is our pleasure to introduce the 2015 Cellular and Molecular Bioengineering (CMBE) Young Innovators. This is the second year of this competition, and we are excited to see the depth and quality of research in the field of Cellular and Molecular Bioengineering continuing to grow.

The corresponding authors in this issue were selected through a competitive process that was initiated in Fall 2014 when self-nominations were solicited from active members of the Biomedical Engineering Society (BMES). The competition is open to all tenure-track assistant professors working in the field of Cellular and Molecular Bioengineering. Applicants were asked to submit a biosketch and an abstract to be considered for the competition. This year's Young Innovators were chosen from a highly competitive pool of candidates. As a result of the selection process, they were invited to submit full-length manuscripts that underwent through a rigorous peer-review process prior to publication in this invited issue. The application pool was outstanding and as a result, the Young Innovators featured here are some of the "best and brightest" working in the field. Notably, 7 of the 11 Young Innovators in 2015 are women.

In the area of cellular engineering, Stephanie Willerth and associates at the University of Victoria developed a system to control the release of retinoic acid to pluripotent stem cells over extended periods. They showed that the incorporation of poly (e-caprolactone) microspheres into cell aggregates induces differentiation into a neuronal phenotype. This approach holds significant promise for neuronal tissue engineering. Deok-Ho Kim at the University of Washington describes the use of nanostructured surfaces to show that human-induced pluripotent stem cell derived cardiomyocytes mimicking a Duchenne muscular dystrophy (DMD) phenotype have an impaired response to microenvironmental structural cues, suggesting these materials may help stratify the cell types to create a better disease model. Shelly Peyton's group at the University of Massachusetts investigated the extracellular factors driving smooth muscle cell migration and proliferation. They found that the complexity of the microenviroment is a key factor driving cell behavior, underscoring the need to create systems that more completely recapitulate the in vivo microenvironment. Anjelica Gonzalez's group at Yale University describes the use of liquid chromatography/tandem mass spec- trometry to analyze the extracellular matrix deposited by pericytes, showing distinct differences between its composition and the ECM deposited by smooth muscle cells. Their results indicate that this matrix supports endothelial cell tubulogenesis, and suggest that it has potential as an engineering scaffold. Brent Hoffman at Duke University used a FRET-based biosensor to demonstrate that vinculin supports tension at cell edges and compressive forces at the center of the cell. This is paradigm-shifting concept as it provides evidence that compressive loads help to maintain focal adhesions. Princess Imoukhuede at the University of Illinois at Urbana-Champaign contributed a paper describing the endothelial receptor numbers VEGFRs, NRP1, and PDGFRs. This work demonstrates there is a cross-talk between receptor classes and lays a solid foundation for the development of more accurate, complex computational models to describe the factors driving angiogenesis.

Several Young Innovators described novel biomaterials designed to control and manipulate cell behavior and/or deliver drugs. Akhilesh Gaharwar and his group at Texas A\&M described a new biomaterial composite which combines poly(ethylene glycol) (PEG) with silicate nanoparticles to create composite microfibers to allow for control over material mechanics and adhesive characteristics. The authors demonstrated the utility of these materials for creating complex multicellular structures, laying the foundation for a class of materials for the controlled presentation of mechanical and chemical cues. Lijie Grace Zhang and associates at George Washington University have developed 3D printed materials which may be used to improve osteochondral regeneration. They used novel complex materials containing organic and inorganic bioactive nano-inks thus controlling roughness, structure, the presentation of bioactive cues, and creating impressive scaffolds which more closely mimic the native environment. Nicole Steinmetz at Case Western Reserve explored the use of viral-based nanoparticles for tumor targeting. Her group investigated the in vivo efficacy of targeted and stealth particles, providing additional insights into the design parameters for selective delivery to tumors. Zhen Gu and associates at the University of North Carolina developed novel hyaluronic acid-based microparticles that selectively release insulin in hyperglycemic environments. Notably, the particles were validated by both in vitro 
and in vivo experiments. These results are truly exciting as they lay a foundation for long-term, self-regulating diabetes treatment, representing a significant advance over the current state of the art. Danielle Benoit's lab at the University of Rochester studied methods of parthenolide delivery using micelles for the treatment of acute myeloid leukemia while overcoming the problem of rapid clearance from the blood. Parthenolide-loaded micelles show high efficacy in vitro, and this work motivates future in vivo studies.

The 2015 CMBE Young Innovators will present their new research at the 2015 Annual Biomedical Engineering Society Meeting in Tampa, Florida. A special two-part platform session will be held at 8 a.m. and 1:45 p.m., on Friday, October 9, 2015 as part of the Cellular and Molecular Bioengineering track, and each corresponding author will present his or her research and be recognized as a 2015 CMBE Young Innovator. We encourage you to attend and learn more about some of the most exciting and innovative research being conducted by these investigators, and interact with the journal on Twitter (www.twitter.com/ CMBEjournal) and Facebook (www.facebook.com/ CMBEjournal).

The 2016 CMBE Young Innovators competition is now underway, with self-nominations due by November 6, 2015. Interested BMES members who hold a tenure-track position at the rank of Assistant Professor (or equivalent) are invited to submit a 200-word abstract and a 2-page NIH-style biosketch to Editor-
in-Chief Michael King at mike.king@cornell.edu. Please consider nominating yourself or encouraging your eligible colleagues to do so. We hope that you enjoy this year's Young Innovators issue, and look forward to communicating more of the most innovative research in CMBE in 2016!

\section{Cynthia A. Reinhart-King}

Department of Biomedical Engineering Cornell University, 302 Weill Hall, 526 Campus Rd, Ithaca, NY 14853, USA

Electronic mail: cak57@cornell.edu

\section{Christine E. SCHMidT}

J. Crayton Pruitt Family Department of Biomedical Engineering, University of Florida, Gainesville, FL 32611, USA

\author{
Nicholas A. Peppas \\ Departments Chemical Engineering, \\ Biomedical Engineering and Pharmacy, \\ The University of Texas at Austin, \\ Austin, TX 78712, USA
}

